\title{
Aperfeiçoamento da produção de partículas de óxido de zinco para aplicação em células solares
}

\section{(Improvement of production of zinc oxide particles for application in solar cells)}

\author{
G. A. R. Maia ${ }^{1 *}$, L. F. G. Larsson ${ }^{2 *}$, A. Viomar ${ }^{3 *}$ E. C. R. Maia ${ }^{4 * *}$, H. de Santana ${ }^{5 * *}$, P. R. P. Rodrigues ${ }^{6 *}$ \\ *Universidade Estadual do Centro-Oeste, Departamento de Química, Rua Simeão Camargo Varela de Sá, n. 03, \\ Guarapuava, PR, Brasil 8504080 \\ **Universidade Estadual de Londrina, Departamento de Química, Rodovia Celso Garcia Cid s/n, CP 6001, \\ Londrina, PR, Brasil 86051-990

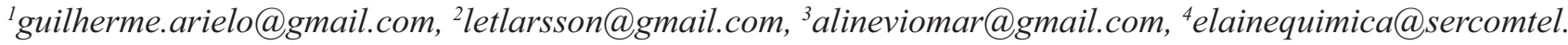 \\ com.br, ${ }^{5}$ hensan@uel.br, ${ }^{6}$ prprodrigues@gmail.com
}

\section{Resumo}

$\mathrm{O}$ óxido de zinco $(\mathrm{ZnO})$ nanoestruturado contém características físico-químicas de elevada importância para utilização em células solares. A padronização e regularidade das dimensões das partículas nanométricas é de difícil obtenção; fatores como natureza do ânion do sal precursor, temperatura de reação e quantidade de água podem interferir no tamanho das partículas. Este trabalho tem como objetivo estudar a influência das variáveis do processo de síntese das nanopartículas de $\mathrm{ZnO}$, visando a aplicabilidade em células solares. Foi aplicado delineamento fatorial incompleto Box-Behnken $\left(3^{3}\right)$, de acordo com o método de coprecipitação, para as três variáveis: concentração de $\mathrm{Zn}\left(\mathrm{NO}_{3}\right)_{2}\left(\mathrm{~mol} . \mathrm{L}^{-1}\right)$, temperatura do meio reacional $\left({ }^{\circ} \mathrm{C}\right)$ e tempo de reação $(\mathrm{h})$. Para caracterização das partículas foram feitas medidas de espalhamento de luz, análise por difração de raios X, caracterização por espectroscopia Raman, microscopia eletrônica de varredura (MEV), aplicação do $\mathrm{ZnO}$ em célula solar com polímero (poli-3-hexiltiofeno - P3HT) e medidas de fotocronoamperometria. A otimização resultou em uma concentração de $\mathrm{Zn}\left(\mathrm{NO}_{3}\right)_{2}$ igual a 0,05 mol.L $\mathrm{L}^{-1}$, tempo de reação de $20 \mathrm{~h}$ e temperatura de $80{ }^{\circ} \mathrm{C}$, onde o menor valor para o tamanho de partícula de $\mathrm{ZnO}$ mostrado pelo modelo foi de $367 \mathrm{~nm}$. As nanopartículas apresentaram cristalito com dimensões de $20 \mathrm{~nm}$, cristalinidade de $82,4 \%$ e fase hexagonal do tipo wurtzita. As imagens de MEV mostraram três tipos diferentes de estruturas devido à variação de concentração do $\mathrm{Zn}\left(\mathrm{NO}_{3}\right)_{2}$. $\mathrm{A}$ amostra de $\mathrm{ZnO}{ }_{370 n m}$ + P3HT + FTO apresentou valor de densidade de corrente (j) de 3,86 mA.cm².

Palavras-chave: polímero, nanotecnologia, delineamento experimental.

\section{Abstract}

Nanostructured zinc oxide ( $\mathrm{ZnO}$ ) has physicochemical characteristics with high importance for use in solar cells. Standardization of the dimensions and regularity of the nanometric particles is hard to obtain, and some factors may interfere in particle size, as the nature of the anion salt precursor, reaction temperature and amount of water. The aim of this work is to study the variables that influence ZnO nanoparticles synthesis, in order to apply in solar cells. Incomplete factorial design Box-Behnken $\left(3^{3}\right)$ was applied, according to coprecipitation method, for three variables: concentration of $\mathrm{Zn}\left(\mathrm{NO}_{3}\right)_{2}\left(\right.$ mol. $\left.\mathrm{L}^{-1}\right)$, reaction medium temperature $\left({ }^{\circ} \mathrm{C}\right)$ and reaction time $(\mathrm{h})$. Particles characterization was made by light scattering measurement, analysis by X-ray diffraction, Raman spectroscopy, scanning electron microscopy (SEM), application of $\mathrm{ZnO}$ in solar cell with polymer (poly-3-hexylthiophene - P3HT) and photochronoamperometry measurement. Optimization results showed the following conditions: concentration of $\mathrm{Zn}\left(\mathrm{NO}_{3}\right)_{2}=0.05$ mol. $\mathrm{L}^{-1}$, reaction time $20 \mathrm{~h}$ and temperature of $80^{\circ} \mathrm{C}$; the lower value for $\mathrm{ZnO}$ particle size showed by the model was $367 \mathrm{~nm}$. The nanoparticles had a crystallite with dimensions of $20 \mathrm{~nm}, 82.4 \%$ crystallinity and wurtzite-type hexagonal phase. The SEM images showed three different types of structures due to the variation of $\mathrm{Zn}\left(\mathrm{NO}_{3}\right)_{2}$ concentration. The sample $\mathrm{ZnO}_{370 n m}+P 3 H T+F T O$ presented current density (j) of $3.86 \mathrm{~mA} . \mathrm{cm}^{-2}$.

Keywords: polymer, nanotechnology, experimental design.

\section{INTRODUÇÃO}

As células solares têm atraído cada vez mais a atenção devido ao baixo custo e ao alto potencial de aplicação, além da capacidade de funcionamento em baixas intensidades de luz. Células solares sensibilizadas são dispositivos de 
conversão de energia solar em energia elétrica que possuem várias interfaces e preferencialmente envolvem a presença de um óxido semicondutor: de acordo com $[1,2]$, o $\mathrm{TiO}_{2}$ é o mais utilizado como eletrodo de trabalho em células solares; outros óxidos semicondutores aparecem como alternativa, entre eles o óxido de estanho, $\mathrm{SnO}_{2}$, e o óxido de zinco, $\mathrm{ZnO}$ [1-3]. O $\mathrm{ZnO}$ tem recebido grande destaque como um material promissor para diversas aplicações, devido à sua alta transparência óptica na faixa do visível e boa condutividade elétrica. É um óxido metálico utilizado na absorção da radiação UV, atua como fotocatalisador, é um semicondutor que possui uma gama de aplicações, entre elas estão fotônica, dispositivos eletrônicos, diodos emissores de luz azul (LED), transistores e células solares [4, 5]. O ZnO possui características físico-químicas que são alteradas de acordo com o método de síntese do óxido [6].

Para a utilização do $\mathrm{ZnO}$ em células solares é necessário que o tamanho de partícula seja em escala de ordem nanométrica [7]. Entre os diversos métodos de síntese que estão sendo apresentados na literatura para a preparação de nanopós de $\mathrm{ZnO}$, pode-se citar o método sol-gel [8], síntese via deposição química [9] e física [10] de vapor, síntese hidrotérmica [11] e o método de precipitação ou coprecipitação [12]. A precipitação de óxidos de metais em solução aquosa é um processo realizado por inúmeras reações em laboratórios e indústrias; ela ocorre de maneira simples pela adição de uma base na solução aquosa do sal do metal [13]. A uniformidade das dimensões das partículas nanométricas esperada é muito exigente; fatores como natureza do ânion do sal precursor, razão de hidrólise, temperatura de reação e quantidade de água podem interferir na composição, morfologia e tamanho destas partículas [13]. Encontrar uma solução aperfeiçoada para um problema de processamento, através da otimização da seleção de um conjunto de fatores potencialmente importantes, nas várias etapas do processo, é tarefa difícil que exige um grande número de ensaios experimentais. Para aumentar a eficiência na busca do aperfeiçoamento de tais problemas, diversas técnicas estatísticas têm sido propostas, particularmente os planejamentos fatoriais. Entre esses, destaca-se a metodologia da superfície de resposta, MSR, que permite desenvolver modelos matemáticos que otimizam a qualidade dos processos [14-17]. Sendo assim, a principal finalidade deste trabalho é estudar a influência das variáveis do processo de síntese do $\mathrm{ZnO}$ : concentração de $\mathrm{Zn}\left(\mathrm{NO}_{3}\right)_{2}$, temperatura do meio reacional e tempo de reação, e caracterizar físico-quimicamente as nanopartículas de $\mathrm{ZnO}$, visando a aplicabilidade em sistemas fotovoltaicos de células solares.

\section{MATERIAIS E MÉTODOS}

\section{Sintese de nanopartículas de $\mathrm{ZnO}$}

As nanopartículas de $\mathrm{ZnO}$ foram sintetizadas pelo método de coprecipitação [18], pela mistura de solução aquosa de $\mathrm{Zn}\left(\mathrm{NO}_{3}\right)_{2} \cdot 6 \mathrm{H}_{2} \mathrm{O}$ (P.A.) e de trietanolamina (TEA)
(P.A.) 0,1 mol. $\mathrm{L}^{-1}$, realizada a $22 \pm 1{ }^{\circ} \mathrm{C}$. A mistura foi deixada em repouso em temperatura e tempo da reação de acordo com o delineamento experimental aplicado (Tabela I). O pó obtido foi centrifugado na velocidade de $4500 \mathrm{rpm}$ e seco em estufa a $100{ }^{\circ} \mathrm{C}$.

Delineamento experimental: para otimizar as condições de controle de tamanho de partícula do $\mathrm{ZnO}$, foi utilizado o delineamento fatorial incompleto Box-Behnken $\left(3^{3}\right)$. Cada variável independente $\left(\mathrm{X}_{1}, \mathrm{X}_{2}\right.$ e $\left.\mathrm{X}_{3}\right)$ foi transformada respectivamente em variável codificada $\left(\mathrm{x}_{1}, \mathrm{x}_{2}\right.$ e $\left.\mathrm{x}_{3}\right)$. Foram realizados 13 experimentos entre estes o ponto central que foi repetido cinco vezes resultando um total de 18 experimentos. As variáveis independentes, concentração de $\mathrm{Zn}\left(\mathrm{NO}_{3}\right)_{2}\left(\mathrm{X}_{1}\right)$, temperatura da reação $\left(\mathrm{X}_{2}\right)$ e tempo de reação $\left(\mathrm{X}_{3}\right)$, foram analisadas em três níveis equidistantes de variação e codificadas com $-1,0$ e +1 (Tabela I), sendo que o tamanho médio das partículas de $\mathrm{ZnO}$ foi escolhido como variável de resposta (Y).

Medidas de espalhamento de luz: as medidas de polidispersão e tamanho médio das partículas de $\mathrm{ZnO}$ foram obtidos via espalhamento de luz, empregando-se um equipamento 90PLUS Particle Size Analizer, Bookhaven, a $25{ }^{\circ} \mathrm{C}$, sendo a água o meio da suspensão, com viscosidade de $0,890 \mathrm{cp}$, ângulo da luz de $90,0^{\circ}$ e $\lambda=659 \mathrm{~nm}$.

\section{Caracterização fisico-química das nanopatículas de $\mathrm{ZnO}$}

Análise por difração de raios $X(D R X)$ : foi feita em um aparelho DRX D2 Phaser, Bruker, com radiação $\mathrm{Cu}-\mathrm{K} \alpha$ de $1,54 \AA$ em $30 \mathrm{kV}, 10 \mathrm{~mA}$ e velocidade de varredura de $0,5^{\circ} \cdot \mathrm{s}^{-1}$ e detector LynxEye. Foi utilizada a equação de Scherrer [19] para calcular o tamanho do cristalito.

Caracterização por espectroscopia Raman: o espectrômetro Raman utilizado foi o Advantage $532^{\circledR}$, DeltaNu, e software NuSpec, excitado em $532 \mathrm{~nm}$ com resolução de $8 \mathrm{~cm}^{-1}$.

Microscopia eletrônica de varredura (MEV): as imagens de microscopia eletrônica de varredura foram realizadas com o MEV VEGA3 da Tescan com detector SE e filamento de tungstênio a $20 \mathrm{kV}$ e WD de $10 \mathrm{~mm}$.

\section{Aplicação das partículas de ZnO: célula solar}

Para a deposição das partículas de $\mathrm{ZnO}$ (eletrodo de trabalho), foi preparada uma pasta com $3 \mathrm{~g}$ de $\mathrm{ZnO}, 0,1 \mathrm{~mL}$ de Triton-X (Vetec), 0,1 mL acetilacetona (Vetec) 99,5\%

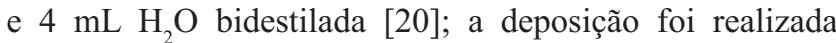
sobre vidro condutor FTO (fluor tin oxide) pelo método de spincoating com velocidade de $3000 \mathrm{rpm}$. Após a deposição o filme foi levado a $450{ }^{\circ} \mathrm{C}$. Como contra eletrodo foi utilizada a platina, $\mathrm{K}_{2} \mathrm{PtCl}_{6}$, eletrodepositada em FTO e o eletrólito foi uma solução de iodeto/triiodeto $\left(\mathrm{I}^{-} / \mathrm{I}_{3}^{-}\right)$[21]. Como sensibilizador (portador de elétrons) da célula solar foi utilizado o polímero poli-3-hexiltiofeno (P3HT) [22].

Medidas de fotocronoamperometria: foram realizadas para avaliar a aplicabilidade das partículas de $\mathrm{ZnO}$ em células solares em medidas de densidade de corrente (j) pelo 
tempo (s). Foram realizadas em um potenciostato Zahner, Zennium Eletrochemical Workstation, acoplado ao XPot e LOT Oriel - Quantum Design GmbH - simulador solar, com lâmpada de $150 \mathrm{~W}$ de xenônio e diâmetro do feixe de 25 $\mathrm{mm}$. A luz foi ligada e desligada no intervalo de tempo de 60 $\mathrm{s}$ durante $750 \mathrm{~s}$, em uma área da célula de $0,25 \mathrm{~cm}^{2}$.

\section{RESULTADOS E DISCUSSÃO}

Polidispersão: as amostras obtidas possuem caráter monodisperso (Fig. 1), sendo o experimento 1 com a menor variação de tamanho das nanopartículas de $\mathrm{ZnO}$. O índice de polidispersão (IP) médio é igual a 0,240 , entretanto as amostras apresentaram uma variação de tamanho com valores de 100 a $1600 \mathrm{~nm}$; quando se trabalha com partículas em escala nanométrica, independente da metodologia utilizada para a produção, o ideal é que todas as formulações apresentem-se monodispersas, com IP igual ou inferior a 0,2 e com diâmetro médio de partícula de $300 \mathrm{~nm}$ [23]; portanto o valor de IP encontra-se adequado considerando a diferença não significativa para o valor obtido; apenas os experimentos 4 e 13 apresentaram valores médios próximos de $300 \mathrm{~nm}$.

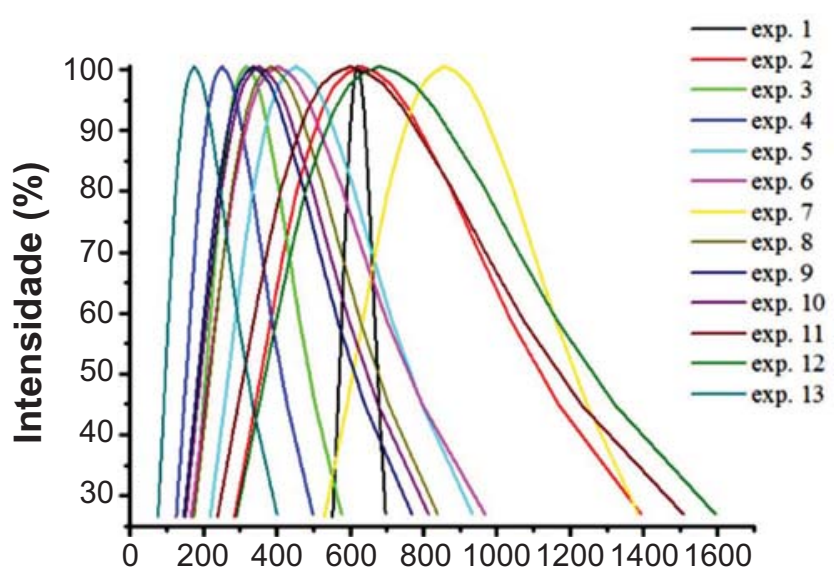

Tamanho partículas de $\mathrm{ZnO}$ (nm)

Figura 1: Distribuição de tamanho de partículas de $\mathrm{ZnO}$ por medidas de espalhamento de luz.

[Figure 1: Size distributions of $\mathrm{ZnO}$ particles by light scattering measurements].

\section{Delineamento experimental}

Para um aperfeiçoamento do processo de produção das partículas de $\mathrm{ZnO}$ foram estudas as variáveis: concentração de $\mathrm{Zn}\left(\mathrm{NO}_{3}\right)_{2}$, temperatura da reação e tempo de duração da reação até o processo de centrifugação e separação do precipitado. A Tabela I apresenta as variáveis independentes codificadas, os níveis de variação em valores originais e as respostas referentes ao tamanho médio de partículas de $\mathrm{ZnO}$, expressas em valores médios de três repetições.

O modelo quadrático, contendo as variáveis independentes codificadas, está representado pela Equação $A$, onde os coeficientes de regressão foram obtidos por $\beta=\left(B^{\prime} B\right)^{-1} B^{\prime} Y$ [24], onde $B$ é a matriz de delineamento
Tabela I - Nível de variação, variáveis independentes codificadas (x) e originais $(\mathrm{X})$ e tamanho médio de partículas de $\mathrm{ZnO}(\mathrm{nm})(\mathrm{Y})$ e desvio padrão $(\sigma)$.

[Table I - Variation level, coded $(x)$ and originals $(X)$ independent variables and average particle size of $\mathrm{ZnO}(\mathrm{nm}) \mathrm{Y})$.]

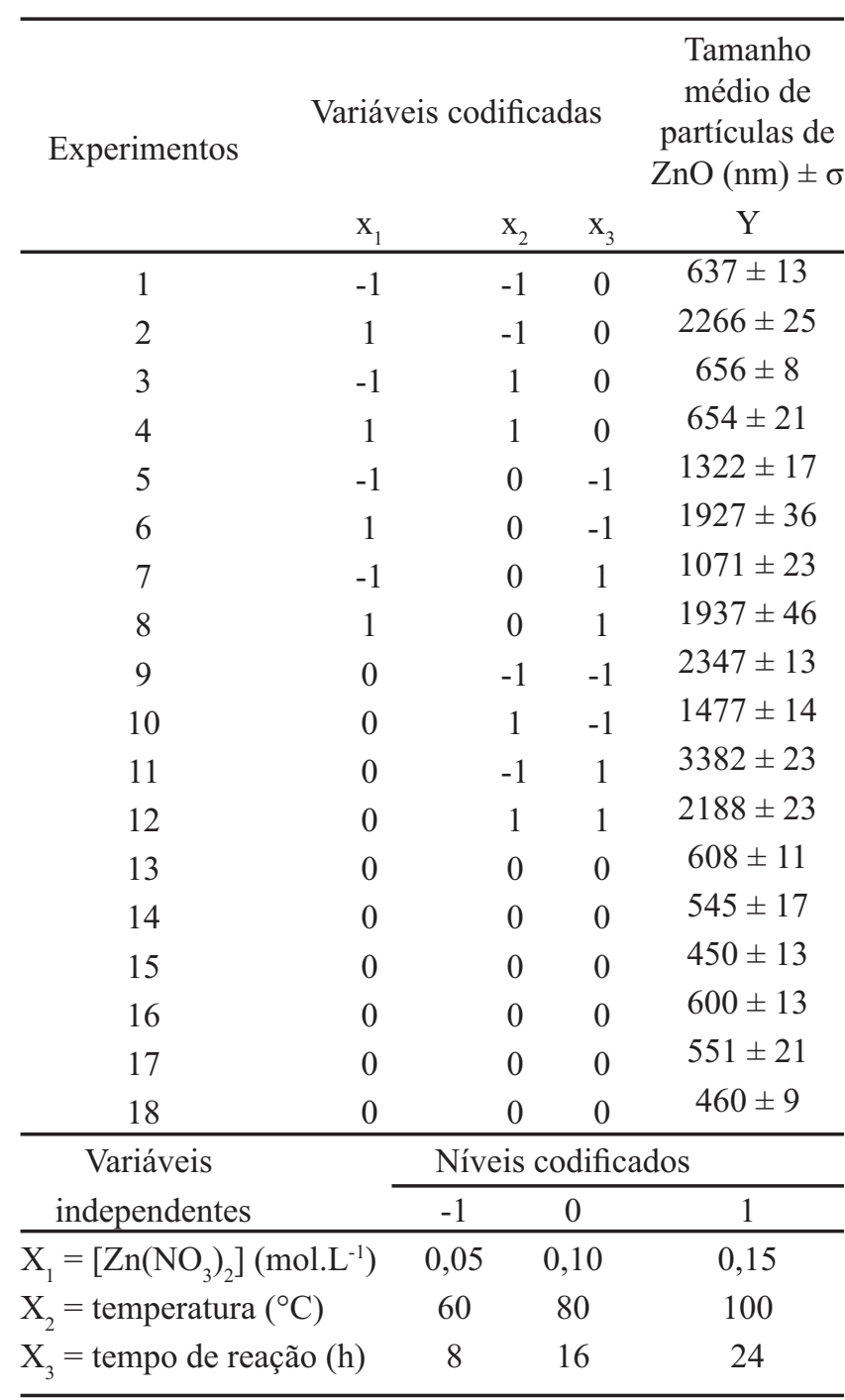

contendo os termos lineares, quadráticos e de interação, e Y é o vetor resposta.

$$
\begin{aligned}
& Y=535,67+387,34 x_{1}-457,19 x_{2}+188,07 x_{3}- \\
& 133,32 x_{1}^{2}+650,98 x_{2}^{2}+1161,85 x_{3}^{2}- \\
& 407,62 x_{1} x_{2}-65,25 x_{1} x_{3}-81,10 x_{2} x_{3}
\end{aligned}
$$

A análise de variância, ANOVA (Tabela II), mostra que o modelo proposto foi significativo em nível de 5\% para todos os termos da equação. Para o teste F, o F calculado $\left(\mathrm{F}_{\mathrm{c}}\right)$ é superior ao $\mathrm{F}$ tabelado $\left(\mathrm{F}_{\mathrm{t}}\right)$ mostrando que o modelo apresentou uma regressão significativa, com os respectivos graus de liberdade. Barros Neto et al. [25] afirmam que uma regressão pode ser significativa do ponto de vista do teste $\mathrm{F}$, mas pode não servir para realizar previsões, uma vez que cobre uma faixa pequena da variação dos fatores estudados. O critério estabelecido por [26], citado em [25], 
Tabela II - Análise de variância (ANOVA) para tamanho médio de partículas de $\mathrm{ZnO}(\mathrm{nm})$ usando o delineamento fatorial incompleto $3^{3}$.

[Table II - Analysis of variance (ANOVA) for average particle size of $\mathrm{ZnO}(\mathrm{nm})$ using the incomplete factorial design $3^{3}$.]

\begin{tabular}{lcccc}
\hline $\begin{array}{c}\text { Fonte de } \\
\text { variação }\end{array}$ & G.L. & $\begin{array}{c}\text { Soma dos } \\
\text { quadrados }\end{array}$ & $\begin{array}{c}\text { Quadrado } \\
\text { médio }\end{array}$ & $\mathrm{F}_{\mathrm{c}}$ \\
\hline Regressão & 9 & 11680606,60 & 1297845,17 & $284,48^{*}$ \\
Linear & 3 & 3155383,64 & 1051794,54 & $230,55^{*}$ \\
Quadrática & 3 & 7817251,31 & 2605750,43 & $571,17 *$ \\
Interação & 3 & 11680606,60 & 3893535,53 & $51,72^{*}$ \\
Resíduos & 8 & 547057,05 & 68382,13 & \\
Total & 17 & & & \\
* Significativo em nivel de 5\%.
\end{tabular}

* Significativo em nível de 5\%

sugere que para uma regressão ser significativa não apenas estatisticamente, mas também para fins preditivos, o valor de $F_{c}$ para a regressão deve ser maior que o valor de $F_{t}$; como mostrado na tabela ANOVA (Tabela II) o valor de $\mathrm{F}_{\mathrm{c}}$ $=284,48$ para a regressão é significativo em nível de 5\%, pois $F_{t}=3,39$, então $F_{c}>F_{t} O$ valor do coeficiente total de determinação observado $\left(\mathrm{R}^{2}\right)$ foi de $95,74 \%$ e a percentagem de variância explicada pelo modelo foi de 90,94, que pode ser considerado adequado, pois, de acordo com [27], para se obter um bom ajuste do modelo aos dados experimentais o valor do $\mathrm{R}^{2}$ deve ser superior a $80 \%$, portanto o valor do $\mathrm{R}^{2}$ e $F_{c}>F_{t}$ mostram que a equação obtida pode ser utilizada para fins preditivos.

A região de combinação binária entre as variáveis originais temperatura da reação $\left({ }^{\circ} \mathrm{C}\right)$ e tempo de reação $(\mathrm{h})$ pode ser observada por meio das curvas de nível apresentadas na Fig. 2. A figura obtida pelo software Statistica ${ }^{\circledR}$ mostra as regiões de contorno da superfície de resposta para a variável dependente, tamanho médio de partículas de $\mathrm{ZnO}(\mathrm{nm})$,

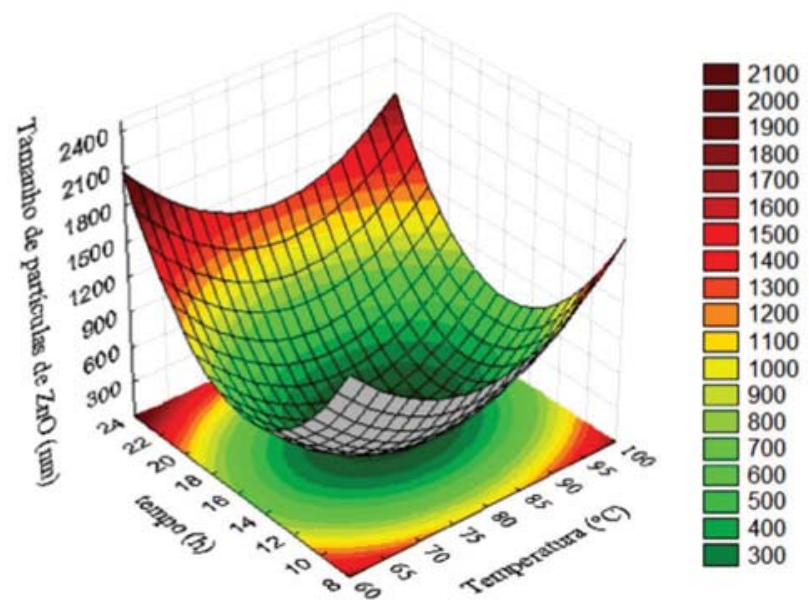

Figura 2: Superfície de resposta para o tamanho médio das partículas de $\mathrm{ZnO}(\mathrm{nm})$ fixando-se a variável $\mathrm{Zn}\left(\mathrm{NO}_{3}\right)_{2}$ em $0,05 \mathrm{~g} . \mathrm{L}^{-1}$.

[Figure 2: Response surface for the average particle size of $\mathrm{ZnO}(\mathrm{nm})$ fixing the variable $\mathrm{Zn}\left(\mathrm{NO}_{3}\right)_{2}$ in $\left.0.05 \mathrm{g.L} \mathrm{L}^{-1}.\right]$

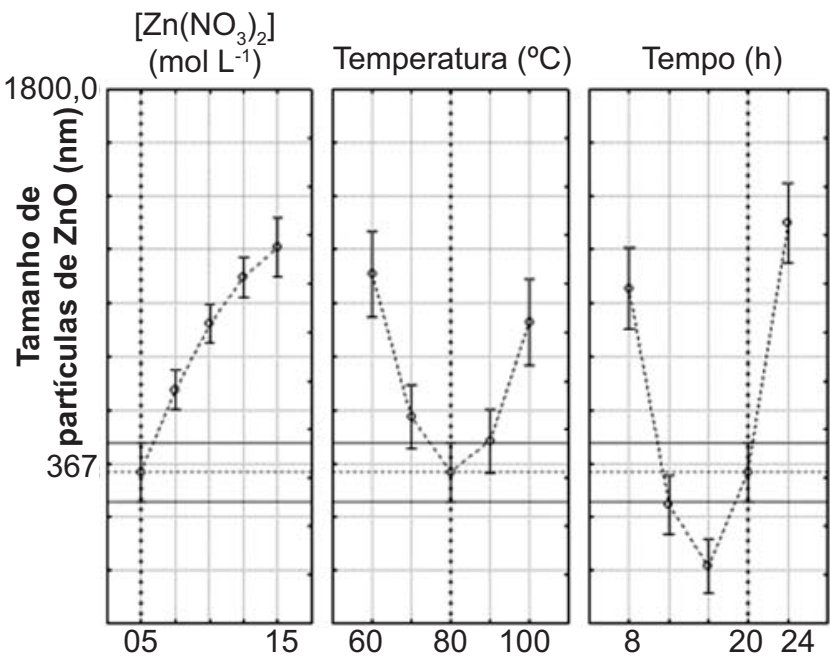

Figura 3: Gráfico das condições ótimas para as variáveis estudadas no processo.

[Figure 3: Chart of optimal conditions for the variables in the process.]

obtidas pelo modelo matemático, fixando-se a variável $\mathrm{X}_{1}$ em 0,05 g.L. $L^{-1}$. Com base na Fig. 2 observa-se que a região onde se estabelece o menor valor de tamanho médio de partícula, situa-se próximo ao ponto central para as duas variáveis $\mathrm{X}_{2}$ e $\mathrm{X}_{3}$, fornecendo como melhor resposta um valor próximo a $300 \mathrm{~nm}$.

A Fig. 3 corresponde ao gráfico de otimização, a fim de minimizar a resposta do tamanho médio das partículas de $\mathrm{ZnO}(\mathrm{nm})$ e apresentar o resultado de cada variável envolvida no processo, onde se observa o nível ótimo para cada uma. Na Fig. 3 obtém-se a configuração de cada fator para a melhor resposta do processo de síntese, onde a concentração obtida de $\mathrm{Zn}\left(\mathrm{NO}_{3}\right)_{2}$ é 0,05 mol.L $\mathrm{L}^{-1}$, sendo este seu limite inferior do delineamento, para um tempo de $20 \mathrm{~h}$ e uma temperatura de $80{ }^{\circ} \mathrm{C}$, onde o menor valor pelo modelo foi um tamanho de partícula de $\mathrm{ZnO}$ de $367 \mathrm{~nm}$. $\mathrm{ZnO}$

Caracterização fisico-química das nanopartículas de

Análise por difração de raios $X(D R X)$ : a $\mathrm{DRX}$ foi realizada para investigar a fase do cristal e o tamanho do cristalito da amostra obtida pela otimização das variáveis do delineamento. Com o resultado de DRX (Fig. 4) é possível afirmar a formação de nanopartículas com fase cristalina do tipo wurtzita, com picos de difração intensos em $2 \theta$ de $31,55^{\circ}, 34,24^{\circ}$ e $36,03^{\circ}$, correspondendo respectivamente aos planos (100), (002) e (101), e picos em $2 \theta$ de 47,27 , $56,33^{\circ}, 62,70^{\circ}, 66,20^{\circ}, 67,69^{\circ}$ e $68,88^{\circ}$, correspondendo respectivamente aos planos (102), (110), (103), (200), (112) e (201), que representam célula unitária no sistema hexagonal compacto característica do $\mathrm{ZnO}$. Os parâmetros de rede obtidos na amostra são $a=3,242 \AA$ e $c=5,195 \AA$, que estão de acordo com [28], referentes ao $\mathrm{ZnO}$ que tem uma célula unitária com dois parâmetros de retículo cristalino $a$ e $c$ na proporção de $c / a=1,633$ (em uma estrutura ideal de wurtzita) e pertence ao grupo de espaço na notação 


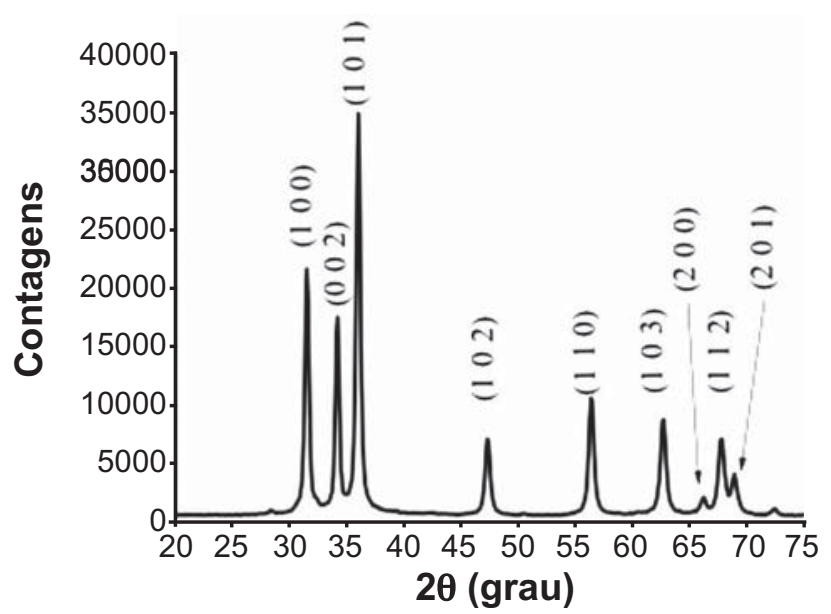

Figura 4: Difratograma de raios $\mathrm{X}$ para as partículas de $\mathrm{ZnO}$. [Figure 4: X-ray diffraction pattern of $\mathrm{ZnO}$ particles.]

Schoenflis e P6 3 me na notação de Hermann-Mauguin. Esta afirmação bem como a catalogação dos picos com os índices de Müller foram feitos utilizando-se de comparação os dados cristalográficos JCPDS 01-075-0576 do software $\mathrm{EVA}^{\circledR}$, referente ao $\mathrm{ZnO}$ puro. Utilizando-se da equação de Scherrer [19], foi calculado o tamanho do cristalito que possui tamanho médio de $20 \mathrm{~nm}$, valor esse que se encontra de acordo com a literatura, que apresenta valores entre 10 e $50 \mathrm{~nm}[8,29,30]$. Não foram detectados picos que não pertencessem ao $\mathrm{ZnO}$, o que indica que estes cristais são de elevada cristalinidade; a amostra apresentou cristalinidade de $82,4 \%$ e livres de impurezas.

Caracterização por espectroscopia Raman: para avaliar parâmetros como ligação, estrutura e os desvios da idealidade cristalina foram realizadas as medidas das frequências dos fônons e a intensidade de espalhamento determinados pela espectroscopia Raman (Fig. 5). Os sinais de Raman são geralmente muito sensíveis à estrutura do cristal, bem como a defeitos na estrutura do cristal. $\mathrm{O}$ espectro Raman obtido mostra que o pico principal dominante é o $\mathrm{E}_{2 \mathrm{High}} \mathrm{em} 437 \mathrm{~cm}^{-1}$, conhecido como o modo de fônons ópticos de Raman ativo, que é o modo característico da fase hexagonal wurtzita do $\mathrm{ZnO}$, sendo o pico relativo à movimentação de $\mathrm{Zn}$ na rede [3133]. Este pico é descrito por meio de um processo multifónon associado a três modos diferentes: um modo $\mathrm{A}_{1}$ dominante, além de uma componente $\mathrm{E}_{2}$ fraca e uma componente ainda mais fraca $E_{1}[34,35]$. O pico em $232 \mathrm{~cm}^{-1}$ é associado ao modo $\mathrm{B}_{\text {tg }}, v\left(\mathrm{~B}_{2 \mathrm{~g}}\right)\left(\mathrm{ZnO}_{4}\right)$, os modos $\mathrm{E}_{2 \mathrm{High}}-\mathrm{E}_{2 \text { Low }}$ em $332 \mathrm{~cm}^{-1}$ e TA + LO em $662 \mathrm{~cm}^{-1}$ são modos característicos do óxido de zinco segundo a literatura $[36,37]$. Os picos de vibração em torno de $537-584 \mathrm{~cm}^{-1}$ são atribuídos aos modos $\mathrm{E}_{1 \text { Low }}$ envolvidos com defeitos estruturais formados por vacâncias de oxigênio, Zn e portadores de carga livres [31,32, 35]; a baixa intensidade desses picos mostra uma pequena densidade de vacâncias de oxigênio. Esse fato é confirmado pela presença de um modo $\mathrm{E}_{2}$ mais forte e de um modo $\mathrm{E}_{1 \text { Low }}$ mais fraco. Na região de maior energia, é possível observar a presença de picos de $1047-1153 \mathrm{~cm}^{-1}$ atribuídos ao modo $\mathrm{E}_{1 \text { Low }}$ associado a overtones e/ou combinação de bandas [31].

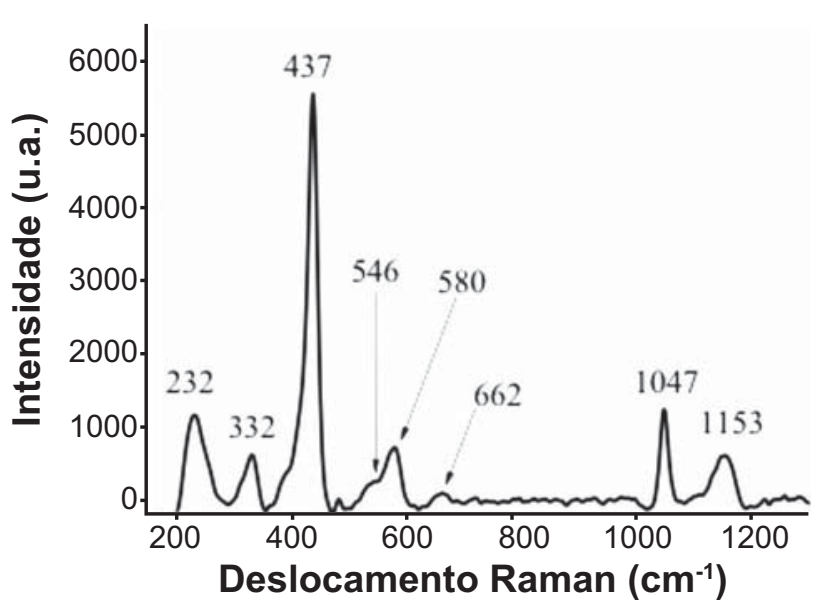

Figura 5: Espectro Raman para as partículas de $\mathrm{ZnO}$.

[Figure 5: Raman spectrum of $\mathrm{ZnO}$ particles.]

Microscopia eletrônica de varredura (MEV): foram realizadas as imagens de $\mathrm{MEV}$ para todas as amostras de partículas de $\mathrm{ZnO}$ do delineamento e estas apresentaram em geral três morfologias diferentes (Fig. 6). A co-precipitação utilizada no presente trabalho refere-se às reações de precipitação que ocorrem em sistemas complexos, onde múltiplas espécies devem ser precipitadas simultaneamente. Na precipitação, o método mais comum para a síntese de nanopartículas é via reações químicas, quando o produto contém apenas um ou dois elementos. No início da precipitação, numerosos cristalitos pequenos se formam (nucleação), mas tendem a se agregar rapidamente (crescimento) para formar partículas maiores e mais estáveis termodinamicamente [38]. As amostras apresentaram variação de suas estruturas de acordo com a concentração do precursor $\mathrm{Zn}\left(\mathrm{NO}_{3}\right)_{2}$, conforme $[39,40]$ que dizem que vários fatores podem afetar o tamanho, a morfologia e as propriedades dos produtos, e que a precipitação pode ser induzida pela modificação de parâmetros relacionados com as condições de tempo de reação, concentração e temperatura da síntese. Para as amostras com menor concentração de $\mathrm{Zn}\left(\mathrm{NO}_{3}\right)_{2}\left(0,05 \mathrm{~mol} . \mathrm{L}^{-1}\right)$ a estrutura gerada foi em forma de esferas, para a concentração de 0,1 mol.L L $^{-1}$ a estrutura apresentou cristais com uma estrutura não definida, e para a maior concentração de 0,15 mol.L $\mathrm{L}^{-1}$ do precursor a estrutura dos cristais foi de prisma-hexagonal (Fig. 6). De acordo com [41], o $\mathrm{ZnO}$ é um cristal com polo positivo $\left(\mathrm{Zn}^{2+}\right)$ e um polo negativo $\left(\mathrm{O}^{2-}\right)$; quando a razão molar é de 1:7,5 as partículas buscam a minimização da energia de superfície e assim as nanopartículas formadas se rearranjam na superfície dos núcleos de $\mathrm{ZnO}$, de modo que as nanoestruturas fiquem com formato de esferas (Fig. 6A). Já quando a razão molar de cátions e ânions é de 1:6 (maior concentração de $\left.\mathrm{Zn}\left(\mathrm{NO}_{3}\right)_{2}\right)$, o complexo $\left[\mathrm{Zn}(\mathrm{OH})_{4}\right]^{2-}$ é formado e tem um caráter preferencial de se adsorver no plano carregado positivamente, o que leva à formação de um prisma-hexagonal alongado ao longo da direção de um só eixo (eixo c), devido à anisotropia particular do seu crescimento (Fig. 6B) [41]. 

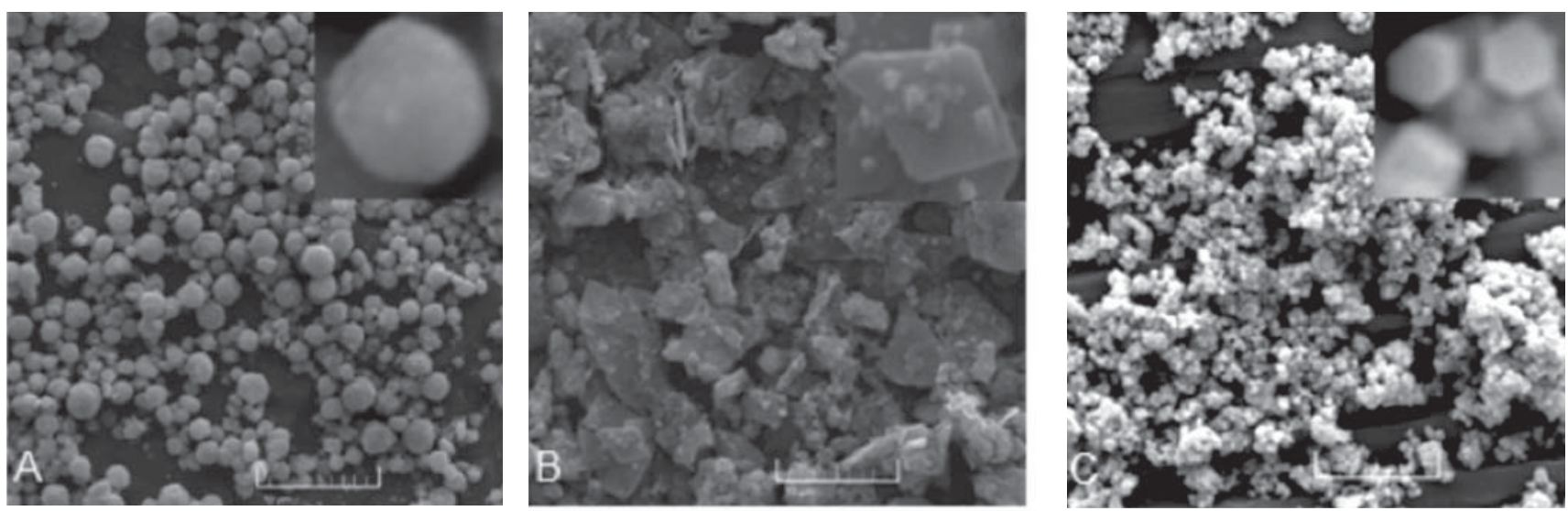

Figura 6: Imagens de MEV para as amostras de $\mathrm{ZnO}$ em forma de esferas (A), sem estrutura definida (B) e de prisma-hexagonal (escala de $10 \mu \mathrm{m})$.

[Figure 6: SEM images of ZnO for samples in the form of spheres (A), with no defined structure (B), and hexagonal - prism (10 $\mu$ m scale).]

\section{Aplicação das partículas de ZnO: célula solar}

Fotocronoamperometria: os resultados das medidas de fotocronoamperometria estão apresentados da Fig. 7. Os resultados mostraram que a presença do polímero (P3HT) nos filmes de $\mathrm{ZnO}$ possibilita a passagem de corrente elétrica e auxilia o material a desenvolver suas propriedades fotovoltaicas na presença de luz e que a diferença do tamanho das partículas proporciona resultados de fotocorrente distintos. A fotoexcitação do polímero adsorvido leva à injeção de elétrons do P3HT no estado excitado para a banda de condução do $\mathrm{ZnO}$. O polímero oxidado é consecutivamente reduzido pelo iodeto (eletrólito), que regenera o P3HT. O fluxo de elétrons injetados no $\mathrm{ZnO}$ segue até o FTO e chega ao contra eletrodo $(\mathrm{Pt})$. Na Pt a redução do triiodeto (eletrólito) fecha o circuito elétrico [20]. As amostras $\mathrm{ZnO}_{1600 \mathrm{~nm}}+\mathrm{P} 3 \mathrm{HT}+\mathrm{FTO}$ e $\mathrm{ZnO}_{370 \mathrm{~nm}}+$ P3HT + FTO apresentaram valores de densidade de corrente de 2,15 e $3,86 \mathrm{~mA} \cdot \mathrm{cm}^{-2}$, respectivamente, na presença de luz; esse aumento do valor de $j$ entre as duas amostras é explicado pela diferença de tamanho das partículas de $\mathrm{ZnO}$, pois segundo $[20,42,43]$, com menor tamanho da partícula novas propriedades mecânicas, elétricas, químicas e ópticas surgem, devido ao aumento da área superficial das partículas, o que possibilita uma maior quantidade do P3HT adsorvido no $\mathrm{ZnO}$; fato este já mostrado por [44], onde para uma célula solar de $\mathrm{TiO}_{2}$ o rendimento aumentou em aproximadamente $8 \%$ em relação ao menor tamanho das partículas do semicondutor. As amostras $\mathrm{ZnO}_{370 \mathrm{~nm}}+$ FTO e FTO, que não receberam o P3HT, não apresentaram mudanças significativas de comportamento elétrico na ausência ou presença de luz; isso foi decorrente ausência do portador de elétrons (P3HT) o que impossibilita o processo de transferência de carga elétrica.

\section{CONCLUSÕES}

$\mathrm{O}$ delineamento experimental empregado neste trabalho mostrou que a melhor resposta para o aperfeiçoamento das

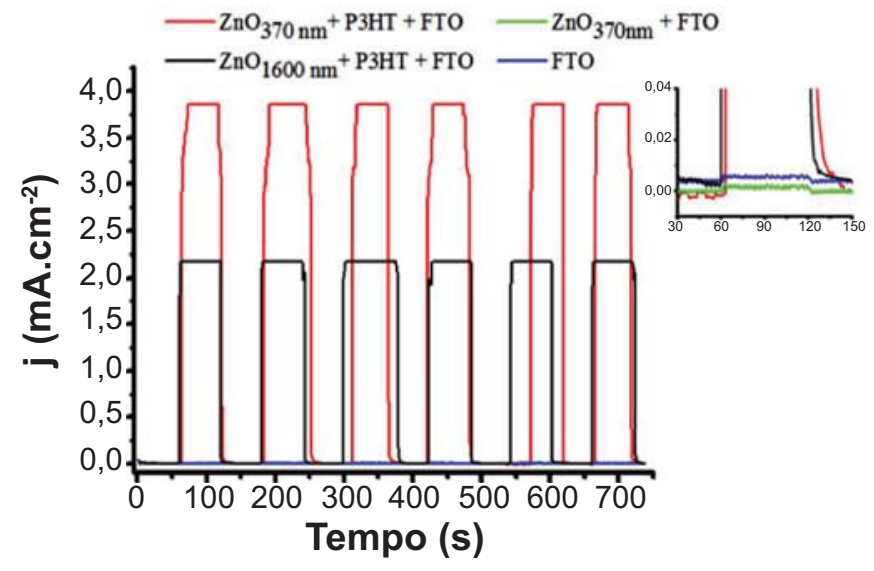

Figura 7: Medidas de fotocronoamperometria para as amostras com luz ligada e desligada no intervalo de tempo de $60 \mathrm{~s} \mathrm{(em} \mathrm{destaque}$ o aumento da escala).

[Figure 7: Photochronoamperometry measurements for samples with light on and off within $60 \mathrm{~s}$ time interval (highlighted the increasing scale).]

variáveis do processo de síntese das nanopartículas de $\mathrm{ZnO}$ foram: $\mathrm{Zn}\left(\mathrm{NO}_{3}\right)_{2}$ de $0,05 \mathrm{~mol} \cdot \mathrm{L}^{-1}$, tempo de $20 \mathrm{~h}$ e temperatura de $80^{\circ} \mathrm{C}$; e o tamanho das partículas de $\mathrm{ZnO}$ obtido pela otimização foi de $367 \mathrm{~nm}$. A análise de difração de raios X apontou que as nanopartículas apresentam elevada pureza, cristalinidade com valor de $82,4 \%$ e tamanho de cristalito de $20 \mathrm{~nm}$. A espectroscopia Raman mostrou os defeitos na estrutura do cristal e a fase característica hexagonal wurtzita do $\mathrm{ZnO}$. As imagens de MEV apresentaram a agregação das nanopartículas e as suas diferentes estruturas. Por meio dos resultados obtidos de DRX, Raman e MEV, é possível afirmar que o método de coprecipitação se mostrou um método de síntese química eficiente na preparação do $\mathrm{ZnO}$ nanométrico e cristalino. Os resultados de fotocronoamperometria mostraram que quanto menor o tamanho das partículas de $\mathrm{ZnO}$ maior será a sua efetividade em conduzir corrente elétrica, podendo aumentar a significativamente a eficiência de células solares. A modelagem utilizando a superfície de 
resposta mostrou ser eficiente e relativamente simples como estratégia de otimização podendo ser considerado de grande utilidade na pesquisa e desenvolvimento de processos de produção e controle de tamanho de partículas para o óxido de zinco para aplicação em células solares.

\section{AGRADECIMENTOS}

À CAPES, ao CNPq, à Finep, à Fundação Araucária e ao Laboratório de Espectroscopia da Central de Multiusuários da PROPPG da UEL.

\section{REFERÊNCIAS}

[1] A.B. Djurisic, A.M.C. Ng, X.Y. Chen, Prog. Quantum Electron. 34 (2010) 191-259.

[2] Y. Tian, C. Hu, X. Wu, Q. Wu, C. Cao, Sol. Energy Mater. Sol. Cells 98 (2012) 83-87.

[3] N.K. Hassan, M.R. Hashim, Y. Al-Douri, Optic - Intern. J. Light Electron Opt. 11 (2014) 2560-2564.

[4] W.L. Suchanek, J. Cryst. Growth 312 (2009) 100-108.

[5] M. Giannouli, F Spiliopoulou, Renewable Energy 41 (2012) 115- 122.

[6] S.M. All-Hilli, M. Willander, J. Nanopart. Res. 8 (2006) 79-97.

[7] U. Ozgur, Y.I. Alivov, C. Liu, A. Teke, M.A. Reshchikov, S. Dogan, V. Avrutin, S.J. Cho, H. Morkoc, J. Appl. Phys. 98 (2005) 11-13.

[8] X. Zhao, M. Li, X. Lou, Adv. Powder Tech. 25 (2013) 372.

[9] P. Chang, Z. Fan, D. Wang, W. Tseng, W. Chiou, J. Hong, J.G. Lu, Chem. Mater. 16 (2004) 5133.

[10] S.C. Lyu, Y. Zhang, C.J. Lee, Chem. Mater. 15 (2003) 3294.

[11] K. Joon-Yub, W.C. Jin, H.K. Sung, Mater. Lett. 65 (2011) 1161.

[12] M. Fan, Y. Jing, D. Yue-Qin, Y. Zhi-Hao, B. Li-Jian, Sens. Actuators B: Chem. 156 (2011) 703.

[13] C. Mayrinck, E. Raphael, J.L. Ferrari, M. A. Schiavon, Revista Virtual Química 6, 5 (2014) 1185-1204.

[14] A.M. Barbosa, E.C. Giese, R.F.H. Dekker, D. Borsato, A. Briones, J. Úbeda, New Biotechnol. 27 (2010) 374-381.

[15] M. Aguirre-Ganzáles, G. Taborda-Ocampo, C. DussanLubert, C. Nerin, M. Rosero-Moreno, J. Braz. Chem. Soc. 22, 12 (2011) 2330-2336.

[16] G.A.R. Maia, S.R.M. Antunes, P.H. Weirich-Neto, S. Borba, R.L. Coppo, D. Borsato, Acta Scientiarum Technol. 36, 4 (2014) 699-705.

[17] M. Tussolini, T. Ichikawa, A.L. Gallina, A. Viomar, A.C. Antunes, M.T. Cunha, E.P. Banczek, P.R.P. Rodrigues, Cerâmica 60 (2014) 417-424.

[18] A.B. Kashyout, M. Soliman, M. El Gamal, M. Fathy, Mater. Chem. Phys. (2005) 230-233.

[19] P. Scherrer, G. Nachrichten, Math. Phys. 2 (1918) 98100.

[20] A.L.A. Parussolo, B.A. Iglesias, H.E. Toma, K. Araki, Chem. Commun. 48 (2012) 6939-6941.

[21] A. Hagfeldt, G. Boschloo, L. Sun, L. Kloo, H. Pettersson,
Chem. Rev. 110, 11 (2010) 6595-6663.

[22] T.N.M. Cervantes, D.C. Bento, E.C.R. Maia, D.A.M. Zaia, E. Laureto, M.A.T. Da Silva, G.J. Moore, H. Santana, J. Mater. Sci.: Mater. Electron. 23, 10 (2012) 1916-1921.

[23] C. Silva, E. Martini, G. Tavares, T. Silveira, M.C. Oliveira, H. Teixeira, Acta Farmacêutica Bonaerense 25 (2006) 17-21. [24] E. Bona, D. Borsato, R.S.S.F. Silva, R. Herrerra, Acta Scientiarum Tech. 22, 5 (2000) 1201-1206.

[25] B. Barros Neto, I.S. Scarmínio, R.E. Bruns, Planejamento e Otimização de Experimentos, Campinas: Editora da UNICAMP, SP, (2003) 299.

[26] G.E.P. Box, J. Wetz, "Criteria for judging adequacy of estimation by an approximate response function", Wisconsin: University of Wisconsin, Tec. Report. (1973) 9.

[27] D. Bisht, S.K. Yadav, N.S. Darmwal, Brazilian J. Microbiol. 44, 1 (2013) 245-252.

[28] T.M. Sabine, S. Hogg, Acta Crystallogr. Sec. B: Struct. Crystallogr. Cryst. Chem. Detail 25 (1969) 2254.

[29] S.S. Silva, F. Magalhães, M.T.C. Sansiviero, Quim. Nova 33, 1 (2010) 85-89.

[30] M.R. Quirino, M.J.C. de Oliveira, D. Keyson, L. Gama, Revista Eletrônica Mater. Processos 9, 2 (2014) 106-111.

[31] R.C. Lima, L.R. Macario, J.W.M. Espinosa, V.M. Longo, R. Erlo, N.L. Marana, J.R. Sambrano, M.L. dos Santos, A.P. Moura, P.S. Pizani, J. Andrés, E. Longo, J.A. Varela, J. Phys. Chem. A 112 (2008) 8970-8978.

[32] M.S. Mohajerani , M. Mazloumi, A. Lak, A. Kajbafvala, S. Zanganeh, S.K. Sadrnezhaad, J. Cryst. Growth 310 (2008) 3621.

[33] S. Gao, H. Zhang, X. Wang, R. Deng, D. Sun , G. Zheng, J. Phys. Chem. B 110 (2006) 15847.

[34] N. Ashkenov, B.N. Mbenkum, C. Bundesmann, V. Riede, M. Lorenz, D. Spemann, E.M. Kaidashev, A. Kasic, M. Schubert, M. Grundmann, G. Wagner, H. Neumann, V. Darakchieva, H. Arwin, B. Monemar, J. Appl. Phys. 93 (2003)126.

[35] C.F. Windisch-Jr., G.J. Exarhos, C. Yao, L. Wang, J. Appl. Phys. 101 (2007) 123711.

[36] R. Cuscó, E. Alarcón-Lladó, J. Ibáñez, L. Artus, J. Jiménez, B. Wang, M. J. Callahan, Phys. Rev. B 75 (2007) 165202.

[37] K.A.J. Pak, Mater. Soc. 4, 1 (2010) 5-9.

[38] Y. Huang, M. Liu, Z. Li, Y. Zeng, S. Liu. Mater. Sci. Eng. B 97 (2003) 111-116.

[39] B.L. Cushing, V.L. Kolesnichenko, C.J. O’Connor, Chem. Rev. 104 (2004) 3893.

[40] U. Manzoor, M. Islam, L. Tabassam, S.U. Rahaman, Phys. E: Low dimensional Systems Nanostructures 41 (2009) 1669.

[41] X. Zhao, M. Li, X. Lou, Adv. Powder Technol. 25 (2013) 372.

[42] G.C. Yi, C. Wang, W.I. Park, Semicond. Sci. Technol. 20, 4 (2005) 22-34.

[43] X. Wang, Y. Ding, C.J. Summers, Z.L. Wang, J. Phys. Chem. B. 108 (2004) 8773-8777.

[44] B. O'regan, M. Grätzel, Nature 353 (1991) 737-740.

(Rec. 02/07/2015, Rev. 10/08/2015, Ac. 10/08/2015) 